\title{
Detection of incipient defects in book bindings. A functional use of the infrared vision combined with optical and nuclear magnetic resonance (NMR) spectroscopy techniques
}

\author{
by S. Sfarra*, M. Tortora ${ }^{\star \star}$, M. Regi ${ }^{\star \star}$, C. Casieri ${ }^{\star \star}$, S. Perilli*, G. Cerichelli** and D. Paoletti*
}

\author{
* Las.E.R. Laboratory, Dept. of Industrial and Information Engineering and Economics (DIIIE), University of \\ L'Aquila, Piazzale E. Pontieri no. 1, I-67100, Monteluco di Roio - L'Aquila (AQ), Italy, \{stefano.sfarra, \\ domenica.paoletti\}@univaq.it; stefano.perilli@graduate.univaq.it \\ ** Dept. of Physical and Chemical Sciences, University of L'Aquila, Via Vetoio (Coppito 1), I-67100, Loc. \\ Coppito - L’Aquila (AQ), Italy, \{mariagrazia.tortora, giorgio.cerichelli\}@univaq.it; \{mauro.regi, \\ cinzia.casieri\}@aquila.infn.it
}

\begin{abstract}
In this study, an ancient book dating back 1822 was inspected by infrared thermography method, working in the active modality. Data was processed by different techniques in order to reduce the impact of non-uniform heating and, in the same time, to improve the thermal imprint of the natural defects detected. In order to confirm the results obtained, both the holographic interferometry and the nuclear magnetic resonance (NMR) techniques were used. The results appear in good agreement among them. Finally, the book binding was also studied into the near-infrared spectrum, while the lateral hinge was inspected by speckle techniques.
\end{abstract}

\section{Introduction}

Within the field of book history, the study of the technical and material aspects of bookbinding constitutes a separate part. As decorative objects, bindings have been studied since the nineteenth century. It was, however, only in the second half of the twentieth century that the history of the technique of bookbinding developed as a discipline in its own right. In addition, the construction of books became a topic of interest. The use of materials, the binding's structure and particular marks of craftsmanship came to be valued as key factors on the book's history. In parallel, the importance of the figure of book conservators grew. Over the last decades, the exchange of information between scientists and conservators has much intensified and has contributing to the study of the physical aspects of books [1-2].

The awareness and recognition that a manuscript or printed book also carries information beyond its text is relatively new, and it has added an interesting dimension to the study of books [3]. The construction and the materials used to make the artefact can reveal valuable data about the historical and social context of a particular text and the book as an object. Through examination of the physical book, information may come to light that could not have been found in another way, for example on the item's provenance [4-6]. In other cases, material evidences may corroborate clues already found through different methods, thus supporting theories that otherwise could remain inconclusive [7-8].

It is widely acknowledged the use of infrared thermography (IRT) method for monitoring defects in book bindings [9-10], parchment sheets [11-12] and, more recently, for the detection of hidden texts in books [13] and mural paintings [14-15]. As shown in the latest two references, the implementation of advanced techniques for processing raw thermograms enhances the results obtained [16]. The present work is focused on the detection of structural problems in books having a rigid and thick front and rear facets; in order to better define the damaged areas to be restored. In particular, the higher order statistics thermography (HOST) [17] and the wavelet transform thermography (WTT) [18] techniques have been selected, since the first one provides a single result that is, therefore, independent by the personal point-of-view of the material scientist, while the second one tends to automatically provide the same advantage thanks to the use of the optimal choice of the scales linked to the complex Morlet wavelet. The more damaged areas, due to a serious woodworm attack, have been pointed out on the Kurtogram result by using a segmentation algorithm described in [16]. The WTT technique worked very well in the confirmation of these suspicious areas when combined with a coherence analysis [19].

However, from the authors point-of-view, in order to in depth clarify the nature of the defects discovered, the thermographic method should be supported by chemical-physical analyses. Indeed, the combined use in a single sensor of IRT and optical non-destructive testing (NDT) techniques, such as holographic interferometry (HI) and/or digital speckle photography (DSP), provided interesting results for the defect detection of natural and artificial defects [20]. In particular, the first one explores in-depth the object to be analyzed, while the second one provides a more superficial evaluation of the state of conservation. On this research field, some experiences have been done in the diagnostics of frescoes [21], panel paintings [22], mosaics [23], marquetries [24], painting on canvas [25] and wooden statues [26]. Therefore, the idea to reproduce the integrated approach also in the case of ancient book bindings naturally set off.

All these types of artworks could also be affected, e.g., by biological damage. Holes in books and bindings, large chewed areas and scraped surface are all evidences of pest attacks. The degree of degradation caused by the 
larvae can be very different. It is linked to the nature of the book binding, the species of insects, the observation time and the building's micro-climate in which they are stored [27].

${ }^{1} \mathrm{H}$ NMR relaxometry proved to be a very effective technique in studying porous systems like paper, allowing the determination of its structure and possible alterations through the different interactions between cellulose and water. In particular, longitudinal $T_{1}$ and transverse $T_{2}$ NMR relaxation times correlation maps characterize non-exchanging water populations [28], and detects both the material's water content and the decay conditions. The use of a single-sided NMR probe offers the chance of non-invasive analyses [29]. Because a surprising matching between the results coming from NMR and IRT techniques has been noticed, it is reasonable to assume that the defective areas are both sub-superficial and structurally dysfunctional. The evidence is provided by the investigation of the book binding (thickness $=3 \mathrm{~mm}$ ) in reflection and transmission modes through a multispectral analysis [30], by working into the near-infrared (NIR) spectrum.

Summarizing, the infestation in books can be controlled both by physical and chemical treatments, but the aim of the security managers of the collections should be to prevent pests just before the trigger of the decay. The integrated approach presented herein follows this direction.

\section{Brief description of the nondestructive testing (NDT) techniques}

Near-Infrared Reflectography (NIRR) and Transmittography (NIRT): introduced in 1968 by J.R.J. van Asperen de Boer [31] as a technique used to look through the paint layers (reflectogram) or to inspect the internal fibres distribution (transmittogram), it became more relevant in the composite materials field in 2013, when a digital subtraction performed in Matlab ${ }^{\circledR}$ environment between the reflectogram and the transmittogram recorded at the same wavelength with the result obtained subsequently analyzed via distance transform (DT), has been applied for the first time [32].

Infrared thermography (IRT): it is a proactive problem-solving and predictive maintenance tool which wields its potential through a thermographer and an infrared camera. Although different techniques useful to process raw thermograms exist [33], the fourth order statistic parameter, namely kurtosis, applied to a square pulse has been investigated. Kurtosis is generally defined as a measure reflecting the degree in which a distribution reaches the peak. In particular, kurtosis provides information regarding the height of the distribution relative to the value of its standard deviation [34]. The surface temperature evolution for a non-defective area after the launching of a square pulse follows a leptokurtic distribution, where the room temperature presents the highest frequency. Therefore, the kurtosis value is very high. For a defective zone, the surface temperature shows a higher or lower room temperature scores depending on the diffusivity value of the defective material. For a defect with a higher thermal diffusivity than the surrounding material, the distribution is more peaked and the kurtosis value is higher for a defective pixel than for a non-defective pixel. On the contrary, the distribution shows a wider peak and the kurtosis value is lower for a defective pixel than for a sound pixel, in a defective material having a lower thermal diffusivity than the surrounding material. At this point, it is possible to estimate the kurtosis values for every pixel in the thermogram matrix and to obtain an image with these values, i.e., a kurtogram. On one hand, the kurtogram provides a first indication of the position of possible sub-superficial defects, and a second indication of their thermal diffusivity [35]. On the other hand, the main benefit of wavelet over Fourier analysis is that both time and frequency localization can be achieved in the former. This is because wavelet analysis employs a wave packet, whereas Fourier analysis uses an infinite wave train of sines and cosines. In the present case the authors use the standard Morlet wavelet, i.e., a Gaussian modulated sine wave of the form [36]:

$$
\psi(n)=\pi^{-\frac{1}{4}} \exp \left(i \omega_{0} \eta\right) \exp \left(-\frac{\eta^{2}}{2}\right)
$$

where, $\pi^{1 / 4}$ is a normalization term, $\eta=n / s$ is the dimensionless time parameter, $n$ is the time parameter, $s$ is the scale of the wavelet, $\omega_{0}=s \omega$ is the dimensionless frequency parameter (taken as $\omega_{0}=2$ in this work), and $\omega$ is the frequency parameter. It should be noted that in wavelet analysis, the wavelet scale is directly related to the oscillation period, and hence to the oscillation frequency. The measuring of phase coherence is carried out to attribute a value of support to measurements of phase difference between two time series (corresponding to defective and defect-free areas), with large values of phase coherence signifying that the phase difference varies smoothly as a function of frequency (i.e., non-random). As such, in [37] is described the wavelet coherency as "an accurate representation of the (normalized) covariance between the two time series". In addition the cone of influence (COI) is the region of the wavelet spectrum in which edge effects become important; it is defined as the e-folding time for the autocorrelation of wavelet power at each scale. This e-folding time is chosen so that the wavelet power for a discontinuity at the edge drops by a factor $e^{-2}$ and ensures that the edge effects are negligible beyond this point.

Holographic interferometry (HI): it provides a means for nondestructive whole-field displacement measurements with very high sensitivity. In double-exposure (DE) HI, two holograms of the two object waves occurring sequentially in time are recorded on a single photographic plate. When such a hologram is reconstructed, two superimposed images of the same object are produced. The interference between these images produces interference fringes overlaid on the image of the object. The interference fringes are indicative of deformation, displacement, rotation and change in refractive index or thickness of the object. When viewing direction is altered, the fringes shift and change their form. $A$ hologram of the object in its initial unstressed state is recorded by exposing the photographic plate. Without removing the photographic plate from the setup, the object is stressed and a second exposure is made [38]. It is also possible to perform the reverse option; it is named ambient drift. 
Digital speckle correlation (DSC): it can measure directly the surface displacement vector and strain tensor to sub-pixel accuracy. This technique involves recording, digitizing and processing a pair of speckle patterns of a sample in different deformation states, one before deformation and another after deformation. If a speckle area in the speckle field before deformation is defined as reference subset and the speckle area corresponding to the subset after deformation is defined as object subset, what is required is to identify the corresponding relation between the two subsets. The difference between the two subsets includes the deformation information of the sample. The method for comparing the two subsets is commonly given by the use of the correlation coefficient [39]. In the present work, the Mat Particle Image Velocimetry (PIV), usually named MatPIV, has been used [40]. It is based on the principles of pattern matching. The displacement of local patterns in an image may be found by calculating the (local) similarity (or minimal difference) between two successive images (specklegrams). Taking into account the small nuances to be detected in the cultural heritage field with respect to the composite material field [41], in this work, a preventive analysis via dark and flat field master images has been used. Indeed, the method can be applied also for speckle imaging by following the experience recently made in X-ray imaging [42].

Nuclear magnetic resonance relaxometry (R-NMR): it represents the loss of magnetization from the spin system to the molecular environment by energy dissipation. The description of simple spin-1/2 systems can be accomplished by means of longitudinal ( $T_{1}$, or spin-lattice) and transversal $\left(T_{2}\right.$, or spin-spin) relaxation times; the values depend on the spin interactions and the geometry of molecular motion. $T_{1}$ and $T_{2}$ change in comparison to the reference systems, which give solid information about materials' structural properties and modifications [43].

\section{Visual inspection of the book binding and historical remarks}

The inspected book having dimensions equal to $0,09 \times 0,165 \times 0,036[\mathrm{~m}]$ and a weight of $\sim 0,6[\mathrm{Kg}]$ is affected by several damages. Indeed, a book's condition is determined by internal factors, namely its constituent materials and structure, and external factors such as storage conditions and handling. Internal factors will determine how well a book ages and how resilient it is to adverse external conditions such as substandard environmental conditions and poor handling. The date of fabrication of the book (1822) coincides with a turning point in the fabrication of this type of artworks. Indeed, during the first half of the nineteenth century paper-making methods in Europe began to change. As the demand for paper increased handmade rag-pulp paper was superseded by machine-made wood-pulp paper. Whilst rag-pulp paper is very stable, machine-made wood-pulp paper is not: it deteriorates far more rapidly, especially in poor environmental conditions. It is important to remark that the sample was bought in a used book stand in Naples (Italy).

In addition, since the codex book form became predominant in Western Europe, many books - as this one have been made with boards larger than the text block. When these books are stored vertically - as in the present case -, gravity gradually pulls the unsupported fore-edges down causing their warp. Eventually, either the text block will fall out of its binding, or the joints between the back and the cover will split. This effect corresponds to the inspected book. In particular, the weaker the structure, the quicker the text block will break off. Hollow spines became prevalent in Western Europe during the nineteenth century. Hollow-spined books are particularly susceptible to damage as the covering material is not adhered to the text block and is likely to tear (Fig. 1a). Spines are not present on the lateral side of the inspected book.

The front facet of the book (Fig. 1b) appears discoloured and stained. All kind of light, whether natural or artificial, causes damage and its effects are cumulative. Ultraviolet radiation causes bleaching, discolouration and the breakdown of organic materials, e.g., flaking of leather, weakening of bookcloth, embrittlement and yellowing of paper (Fig. 1c). The book binding also appears infested by pests, since a diffuse series of small holes affect it completely (Fig. 1b). Usually, the insect infestations become evident with a high (above 65\%) level of relative humidity $(\mathrm{RH})$.

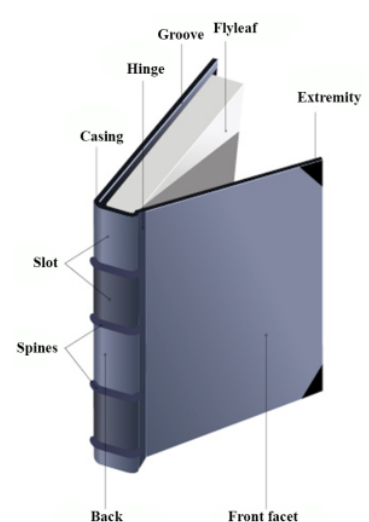

a)

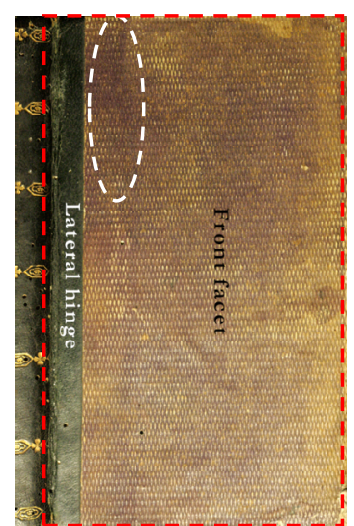

b)

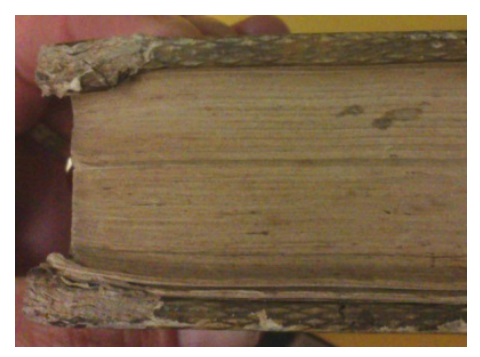

c)

Fig. 1. a) Main parts of an ancient book, b) Photograph of the front facet which includes the lateral hinge of the inspected book. The red dotted rectangle indicates the region of interest (ROI), and c) the lateral view.

In addition, at this level starts the mould growth, the increase in the rate of chemical degradation of paper by acid-catalysed hydrolysis, the corrosion of iron gall inks (it was the most common type of ink in Europe from the eleventh 
Century to the early twentieth Century), and the increase evidence of foxing (it was thought that foxing is caused by metal impurities in paper and microbiological activity) [44]. Finally, the cover materials are different. Around the first half of the nineteenth century, book cloth, buckram, plastic upholstery material, canvas and leather are in use. In the present case, the front facet appears to be made in buckram, while the lateral hinge seems to be made of leather-looking paper (Fig. 1b). Buckram is applied to the book with either non-warping or water-base paste. Buckram is very strong and durable [45].

\section{Results and discussion}

A combined inspection between DSC and IRT methods has been performed through separate devices with their own systems. Deformation maps (from specklegrams) and raw thermograms requested to be re-sampled for correlation purposes. The heating phase (10 minutes), as well as the frame rate ( 1 image every 2 seconds) connected the acquisition system through a timer (Fig. 2). The lamps with a power equal to 250 [W] were installed 0.34 [m] far with respect to the front facet of the book binding. Instead, the thermal camera (operating in the long-wave IR spectrum, 7.5$13 \mu \mathrm{m}$, FLIR S65 HS) and the CMOS camera $(22.2 \times 14.8 \mathrm{~mm}$ at 10 megapixels $-5.7 \mu \mathrm{m}$ pixel pitch) sensor, at 0.72 [m] and 0.66 [m], respectively. The lamps were positioned at $45^{\circ}$ angle. First of all, the cold images (i.e., the first specklegram and the first raw thermogram) have been recorded at the steady-state condition (ambient temperature $=23$ ${ }^{\circ} \mathrm{C}, \mathrm{UR}=32,7 \%$ ). In addition, also the visible image of the book binding has been collected. Subsequently, the lamps were switched on together with the registration of the raw thermograms. At the maximum of the heating phase, the lamps were switched off, the laser $(\lambda=532 \mathrm{~nm}$ as fundamental wavelength, and $250 \mathrm{~mW}$ of power) illuminated the sample one more time, and the registration of the specklegrams started. Obviously, the acquisition of the specklegrams and the raw thermograms continued in parallel along the entire cooling phase (14 minutes). At the end of the process, 720 raw thermograms and 420 specklegrams were collected.

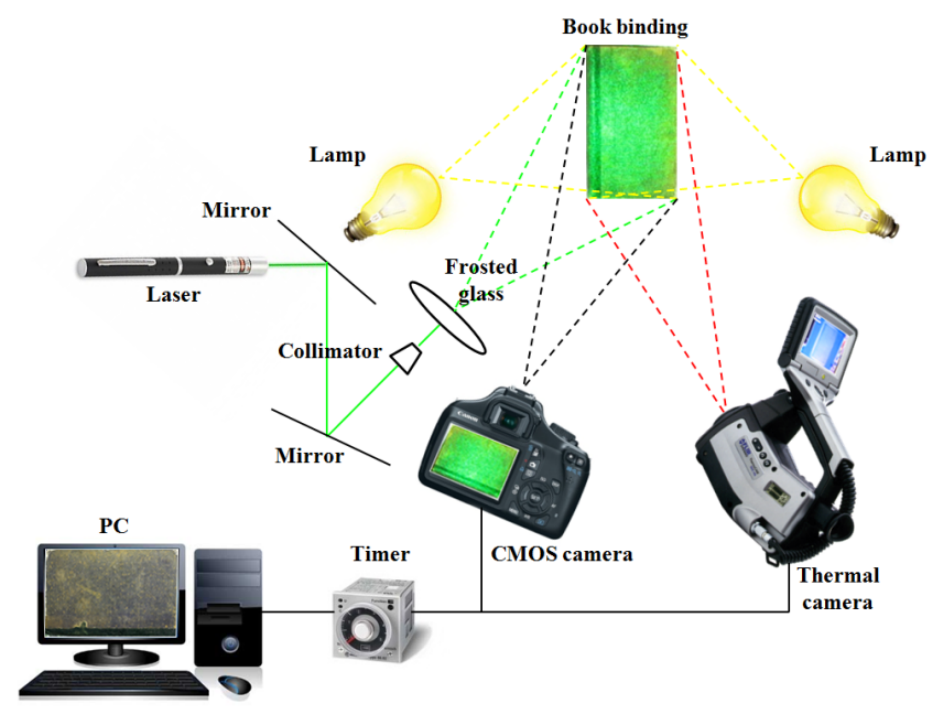

Fig. 2. Synergistic DSC and IRT experimental setup

Concerning the image processing of the raw thermograms, a discrete Fourier transform (DFT) analysis has been conducted [46], involving all pixels of the region of interest (ROI), i.e., the front facet of the book binding. A total of 419 raw thermograms, at $2 \mathrm{~s}$ sampling period, have been acquired during the cooling phase. Therefore, the frequency resolution of the DFT of each pixel thermogram, is $1 / 838 \mathrm{~s}=0.0012 \mathrm{~Hz}$. The first results inherent to the FT at the lower frequencies are not influenced by instrumental noise (Fig. 3a,b); they provide an interesting result of the thermal anomalies. At the higher frequencies, the instrumental noise increases. After the DFT analysis, the raw thermogram linked to $f=1.19 \mathrm{mHz}$ has been selected in order to mark the points to be compared through the wavelet coherence analysis (Fig. 3c). The intent is to establish the best scale which improves the difference between thermograms corresponding to defective area (x) and defect-free area (+) (Fig. 3c). The scale factor $w_{0}=2$ has been chosen for the wavelet Morlet [47]. The average wavelet cohereograms were calculated between all the selected points, both in quadratic amplitude $\left(\mathrm{r}^{2}\right)$ (Fig. 3d) and in phase difference $(\varphi)$ (Fig. 3f). It is possible to observe that the suitable scale $(--$ -) is equal to 8. Finally, a diagnostics concerning a dynamic evolution both of the amplitude and of the phase of the WT was performed which takes into account the COI, drawn by white lines in Figs. 3d,e. The most interesting result linked to the WT phase is represented in Fig. 3f, that corresponds to approximately the eightieth second during the cooling sequence. 


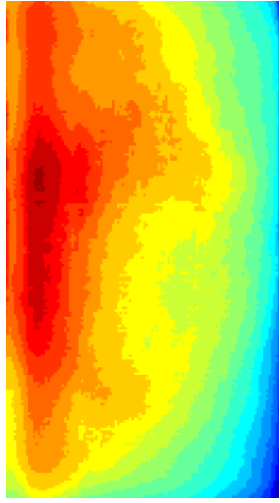

a)

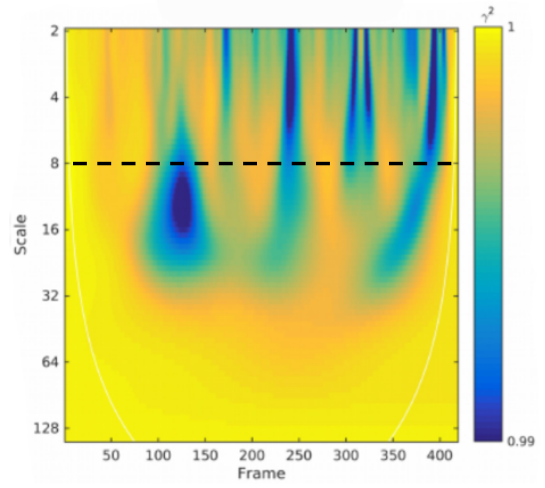

d)

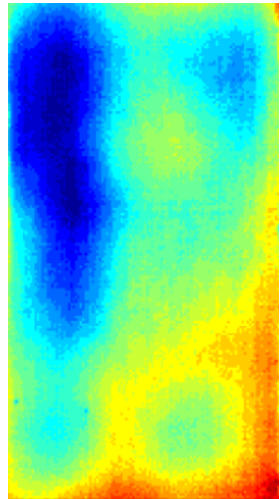

b)

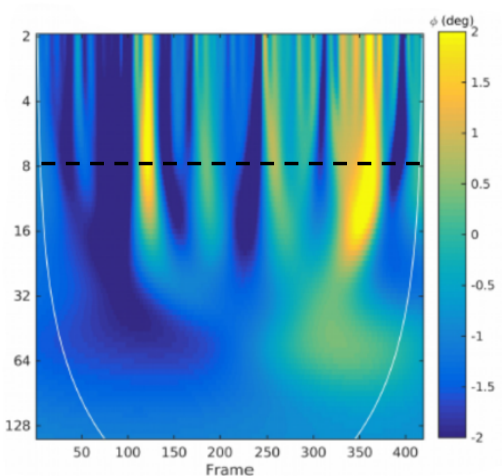

e)

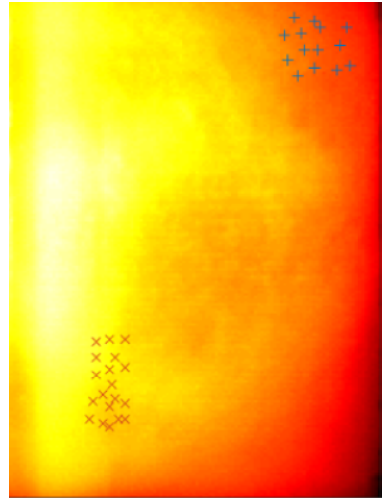

C)

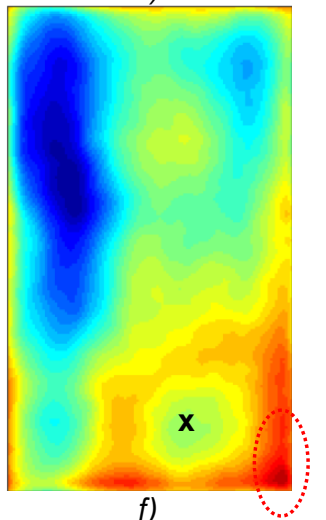

f)

Fig. 3. a) DFT (amplitudegram) at $1.19 \mathrm{mHz}$, b) DFT (phasegram) at $1.19 \mathrm{mHz}$, c) raw thermogram equal to $f=1.19 \mathrm{mHz}$; with +, a defect-free part of the book binding is indicated, while with $\mathrm{x}$, a defective area of the book is pointed out, d) quadratic amplitude cohereogram, e) phase difference cohereogram, and f) WTT result

In order to better differentiate the defective areas with respect to the defects-free areas, additional tests have been conducted. Among these, one variation of $\mathrm{HI}$ was applied, namely, DE technique (Fig. 4a). The two holograms were recorded on the same fine-grained green sensitive holographic plate (VRP-M) made by Slavich ${ }^{\circledR}$, each one capturing the sample in a different state separated by a fixed time interval (2 minutes). The grain size in the emulsion was in the order of $0.03-0.08 \mu \mathrm{m}$. Holographic plates used had dimensions of $10.2 \times 12.7 \mathrm{~cm}$. A G4plus 250 laser by Elforlight Ltd. was used, while the holograms were recorded in ambient drift. In particular, by using an electric heater, the ambient temperature of the super-compact-mini temporary holo Las.E.R. Laboratory was raised up to $29{ }^{\circ} \mathrm{C}$ [48]. It is possible to understand how the increase of the surface temperature on the book binding was very small.

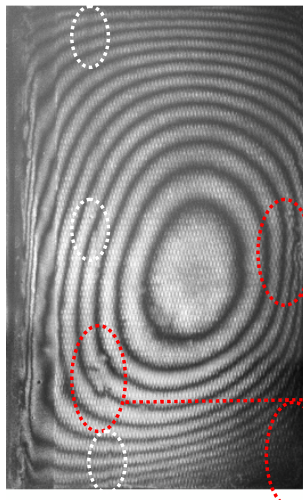

A
B

a)

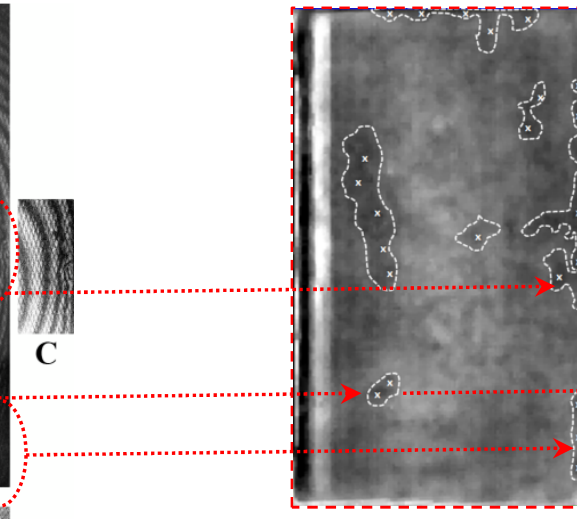

b)

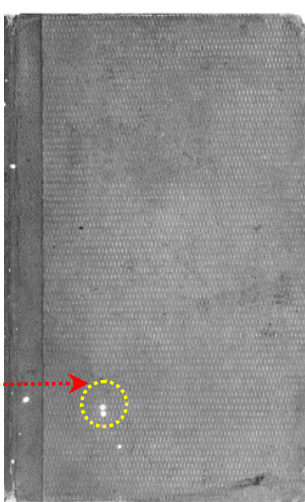

c)

Fig. 4. a) double-exposure (DE) HI result with marked the more evident (A, B, C) sub-superficial defects, b) Kurtogram segmented through a specific algorithm, and c) subtraction of images performed in Matlab ${ }^{\circledR}$ environment between the reflectogram and the transmittogram acquired at $1000 \mathrm{~nm}$ 
The interferogram shown in fig. $4 \mathrm{a}$ was obtained with the first exposure ( $t_{\exp }=3 \mathrm{~s}$ ) $10 \mathrm{~s}$ after switching off the electric heater. Also the second $t_{\exp }$ lasted $3 \mathrm{~s}$, while the initial ambient temperature was, also in this case, equal to $\mathrm{T}=23$ ${ }^{\circ} \mathrm{C}$ [49]. The cusps of fringes indicating damages due to conservation problems and biological attacks, are identified in Fig. $4 a$ by dotted red ovals. Enlargements of these, coming from other interferograms collected in order to confirm the assumption of the damage, are reported on the lateral side of Fig. 4a. They are called A, B and C defects. Particularly interesting are the other anomalies surrounded by three dashed white ovals. Indeed, they are more or less aligned along the same line which is parallel to the major axis of the book binding. It is practically indiscernible to the naked eye one of these anomalies in the visible image shown in Fig. 1b. However, by considering their positions, a possible explanations could be the presence of a sub-superficial stapling which joints together the buckram and the leather parts.

By using the same set of raw thermograms processed in the WTT, a Kurtogram result has also been obtained (Fig. 4b). A superimposition with an automatic extraction of defects via map [50], obtained thanks to an algorithm thought to infrared images, integrates the result. This procedure was necessary in order to find a correlation with the NMR results subsequently explained. The algorithm was developed in two steps. Firstly, the locations of the defects (seeds) must be found. Labeling of the pixels was based on the distance. Secondly, a specific threshold will be obtained for each of the defects detected by region-growing around those seeds. The found thresholding criterion for seed was $T=\alpha \times(\mu+\alpha \times$ $\sigma)$. In practice, the defect shape is grown by gradually decreasing the threshold until a sudden increase in the number of pixels agglomerates together, or an image boundary is encountered [50].

A positive correlation between the interferogram (Fig. 4a) and the Kurtogram (Fig. 4b) can be found. Readers should compare the damaged areas surrounded by red dotted ovals and linked between them by arrows. In addition, the bottom right hand part was also detected by WTT (Fig. 3f). Readers should also notice how the greatest part of the damaged areas is localized on the upper and right borders. Presumably, it could be an index of the favorite position of conservation of the book above a support in the course of time. Part of the damage is also visible in Fig. 1c. Intriguing is also the fact that the same type of material (i.e., the leather) reacts in the opposite way after an HOST analysis. Indeed, a part of the lateral hinge (Fig. 1b) is bright, while the outer limit (i.e., the lateral hinge) is dark (Fig. 4b). Presumably, the overlap between the sheets of different materials on the part of the lateral hinge which appears as bright, i.e., its thickness with respect to the part of the lateral hinge which appears as dark, plays an important role in the detection of that zone. Readers should compare the inspected area surrounded by a dotted red rectangle both in Fig. $1 \mathrm{~b}$ and in Fig. $4 \mathrm{~b}$. In addition, it is possible to notice how the selected points during the WT analysis and inherent to defective and defect-free areas of the book binding are in good agreement with the Kurtogram result (Fig. 4b). In particular, the defective points $(\mathbf{x})$ are located above a defective zone, while the defect-free points $(+)$ are positioned between three distinct defective zones on the upper right hand part.

The digital subtraction of images between the reflectogram and the transmittogram shown in Fig. 4c clarifies how many holes created by wood worms through the front facet of the book binding along the whole thickness.

In particular, six holes appear equally distributed between the buckram and the leather materials, although some very small holes can be detected in the leather part. Particularly of interest are the contiguous holes surrounded by a dotted yellow circle in Fig. 4c, because they are linked to the DSC results shown in Fig. 5. Both the interferogram (Fig. 4a) and the Kurtogram (Fig. 4b) point out how this damage is much more extended in the sub-superficial part of the book binding. The subtraction of images also enhances the detection of dark zones which could be linked to the discolouration of the external layer due to continuous handling.

The different behaviour under a thermal stress of the inspected materials of the book binding (i.e., the leather and the buckram), also in terms of adhesion between superimposed layers, it is clarified through the DSC technique. In this case, the specklegrams have been treated by flat-field calibration and dark subtraction, in order to compare the results obtained after the image processing by MatPIV. In particular, Fig. 5a shows the DSC result without the application of the calibration, while Fig. $5 \mathrm{~b}$ shows the DSC result with the application of the calibration.

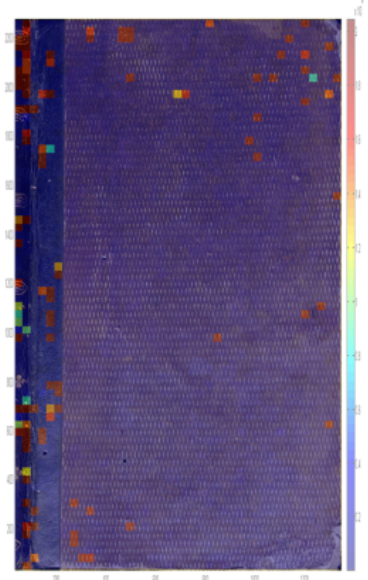

a)

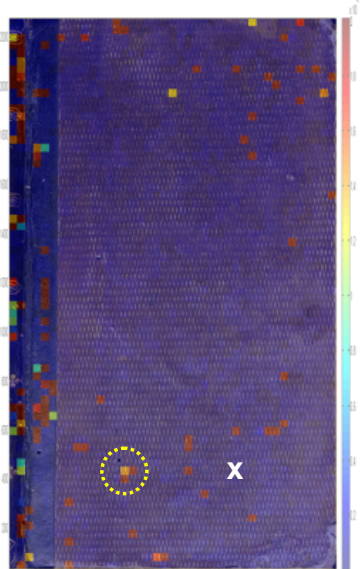

b)

Fig. 5. a) DSC result without pixels correction (image fusion), and b) DSC result with pixels correction (image fusion) 
The calibration procedure consists in a flat-field correction of the image sequence. Usually, this technique is used in the astrophysical context for images recorded by means of a charge-coupled device (CCD) [51]. Unfortunately, in the present case, each image consists in a compressed version of raw image (i.e., JPEG), that contains modified information on the CMOS response to the incoming photons. However, it can be applied to CMOS's images. Flat fielding is the part of the calibration that mitigates the effect of the CMOS's pixel-to-pixel variation in sensitivity, vignetting in the optical system, and shadows created by dust on the components of the imaging system.

Let's imagine a perfect imaging system without hot or cold pixels, dust, and vignetting. If this hypothetical setup is exposed to a uniform light source, then every pixel in this image should have the same analog digital unit (ADU) or pixel value after exposure. Unfortunately, $\mathrm{CMOS}_{\mathrm{s}}$ are not perfect, so pixel values in this "flat-field" image will vary. Just how much they vary tells us exactly how to modify each pixel in the image of a suitable target to correct for the imaging setup's imperfections. In the present case, a total number of sixty dark images, and sixty white images have been recorded. For every image (separated by a fixed time interval each other), the ambient temperature $\left({ }^{\circ} \mathrm{C}\right)$ and the relative humidity (\%) were registered by using a thermohygrometer.

The entire calibration process can be summarized succinctly by the following relationship:

$$
C=\frac{(R-D)}{(F-D)} \cdot m=(R-D) \cdot G
$$

where, $R$ is a raw image, $D$ is the dark master, $F$ is the flat field master, $m$ is the median value (as in the present case) or the mean value of $F-D, C$ is the calibrated image, while $G=\frac{m}{F-D}$ is the gain. The equation's numerator is simply the raw exposure of a suitable target corrected for any thermal "dark" signal due to non-photon-induced electrons in the CMOS. To understand the denominator, think back to the perfect CMOS, where each pixel responded perfectly to the even illumination and needs no correction (in which case the denominator would have a normalized value equal to 1). Assuming the light source is perfectly uniform, the mean of all the pixel values in the flat-field image is a good approximation of the uniform pixel value from the perfect CMOS. If a pixel value in the flat-field image is below the mean value, then it is "underreporting", while any pixels that have values above the mean are "overreporting" [52]. By dividing the flat-field pixel value by the mean of all the pixel in the flat-field image, the normalized pixel value is obtained. A pixel that has a value equal to the mean will be normalized to 1 , while a pixel that has a value less than the mean will be normalized to less than 1 , and pixels with values above the mean will be normalized to more than 1 . The Matlab ${ }^{\circledR}$ script written ad hoc takes care of this normalization automatically. It should now be apparent that master dark frames must be applied to both the raw image and the flat-field image in order for the pixel to be calibrated properly. Failure to subtract the dark current from a flat-field frame will make the denominator incorrectly large, resulting in an improper correction to the raw image. In Fig. 6a, the dark master corresponds to the mean value of the dark images, while the flat field master is the mean value of the flat images. In addition, an example of calibration of the flat images inherent to the present work is reported in Fig. $6 b$.
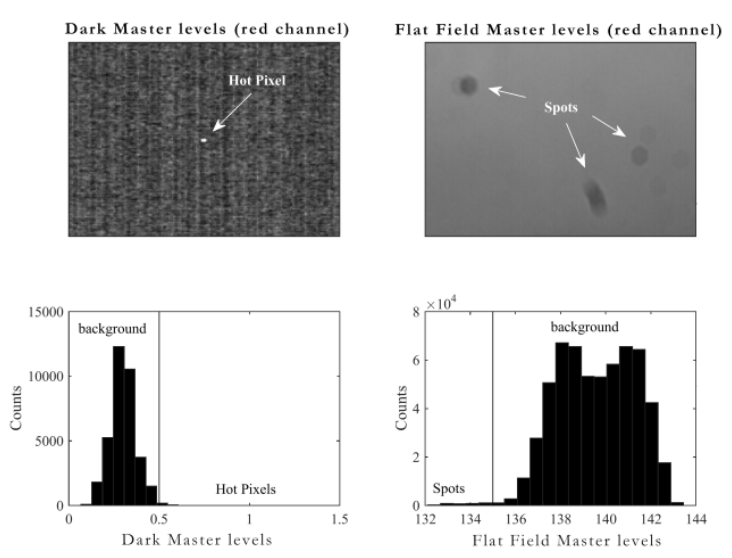

a)
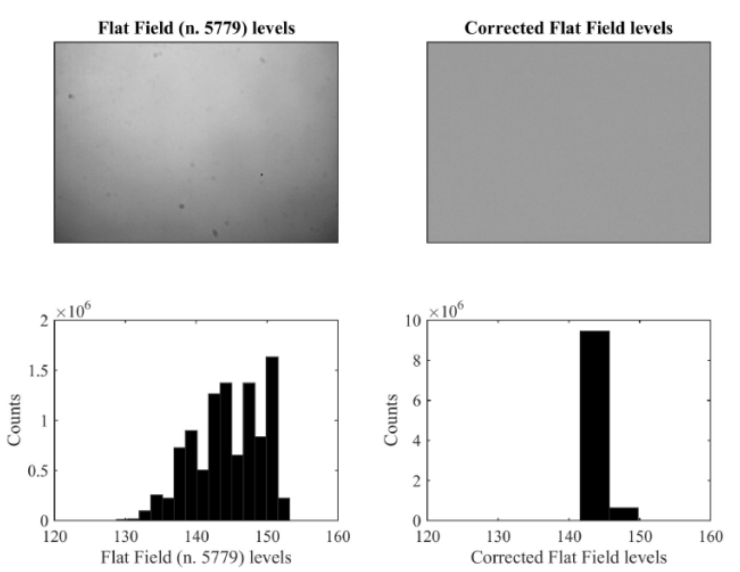

b)

Fig. 6. a) Hot pixels and spots due to dusts or defects of the lens, and b) an example of calibration of a flat image

On the left part of Fig. 6a, an example of badpixel is shown. A badpixel can be defined as an anomalous pixel behaving differently from the rest of the array. For instance, a dead pixel remains unlit (black) while a hot pixel is permanently lit (white). In any case, badpixels do not provide any useful information and only contribute to deteriorate the image contrast. The value at badpixel locations was replaced by the average value of neighboring pixels [53]. In addition, the left side of Fig. $6 \mathrm{~b}$ contains some spots which are magnified on the right side of Fig. $6 \mathrm{a}$, and subsequently corrected on the right side of Fig. 6b. It is also possible to observe that on the right side of Fig. $6 \mathrm{~b}$ the vignetting was normalized. Vignetting is another source of noise on CMOS camera that causes a darkening of the image corners with respect to the 
image center due to limited exposure. Vignetting depends on both pixel location and temperature difference with respect to the ambient. For this reason, $T_{a m b}$ has been recorded for every pixel.

Finally, all the raw specklegrams were normalized, while the specklegrams which provide the greatest in-plane deformation were correlated and displayed in Fig. 5a,b thanks to an image fusion with Fig. $1 \mathrm{~b}$.

The need to correct the specklegrams is due to the fact to eliminate any misunderstanding about false defects. In fact, hot pixels, spots, could be confused with defects of the book binding.

The largest in-plane mechanical deformation is appreciated on the leather material, for which the effect of temperature on the volume was initially studied in [54]. It was found that the average coefficient of cubical expansion calculated for dry materials is equal to $540 \times 10^{-8} /{ }^{\circ} \mathrm{C}$. Another interesting zone is the upper right hand part of the front facet of the book binding, which corresponds to the thermal anomaly revealed by the WTT technique (Fig. 3f). In addition, it is very interesting to notice how after the calibration phase, the two holes detected in the NIR spectrum (Fig. 4c) become evident. In both images, they are surrounded by dotted yellow circles. Finally, it was surprising to see how in the calibrated image, which was processed by MatPIV (Fig. 5b), the contours of the persistent thermal anomaly marked by " $x$ " in Fig. 3f, appears. The points which constitute the ideal contours of the suspicious zone, correspond to the highest variability in terms of in-plane deformation, as it is possible to observe from the rainbow scale of values. For a sake of clarity, the same sign (i.e., " $x$ ") is also reported in Fig. $5 b$ in order to identify the zone of interest.

It is important to underline how, this thermal anomaly takes a part of the $L_{12}$ zone (Fig. 7a) which corresponds to one the zones at higher intensities in the NMR measurements subsequently explained.

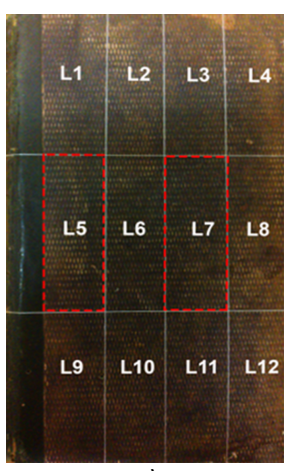

a)

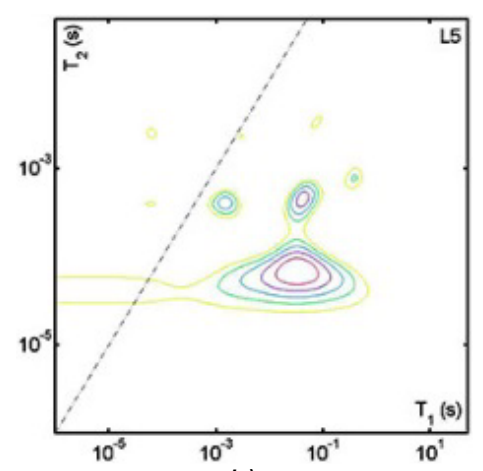

b)

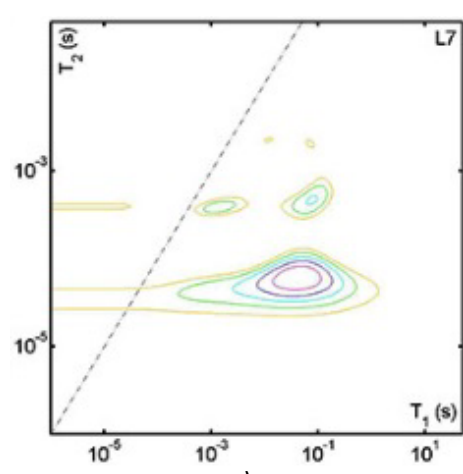

C)

Fig. 7. a) Photograph of the front facet with marked the $L_{1}-L_{12}$ sections for NMR acquisitions, $L_{5}$ and $L_{7}$ (surrounded by red dotted rectangles) have the highest intensities, b) $L_{5}$ section, and c) $L_{7}$ section $N M R T_{1}-T_{2}$ correlation maps

As anticipated in the introduction section, the analysis of the book binding was performed by single-sided ${ }^{1} \mathrm{H}$ NMR correlation relaxometry. In the present study, longitudinal $T_{1}$ and transverse $T_{2} N M R$ relaxation times correlation maps, $T_{1}-T_{2}$, were realized: since the probe is able to measure only the $T_{2}$ values larger than about $70 \mu s$, it can reasonably be excluded that protons different from those belonging to water may appreciably be detected. The NMR measurements have been performed by a single-sided ${ }^{1} \mathrm{H}$ NMR probe [55] (Mq-Profiler, Bruker), working at a Larmor frequency of $17.8 \mathrm{MHz}$ and with a sensitive xyz volume of about $2 \times 0.2 \times 0.8 \mathrm{~cm}^{3}$. The book was previously conditioned at $50 \%$ R.H. and $23{ }^{\circ} \mathrm{C}$ for seven days. The book binding was subdivided into 12 sections according to the rf coil's dimensions $(5 \times 2,5 \mathrm{~cm}), \mathrm{L}_{1}-\mathrm{L}_{12}$ (Fig. 7a) and each section was analyzed in order to compare NMR data with those obtained with the previous analytical techniques.

The pulse sequence used for $\mathrm{T}_{1}-\mathrm{T}_{2}$ maps was [56]:

$$
\left[\text { saturation }-t_{s}-90^{0}-\frac{t_{E}}{2}-\left(180^{0}-\frac{t_{E}}{2}-\text { acquisition }-\frac{t_{E}}{2}\right)_{n}-\tau_{R D}\right] m
$$

where, $\tau_{\mathrm{RD}}=2 \mathrm{~s}$ is the recycle delay time, $t_{\mathrm{E}}=44 \mu \mathrm{s}$ is the echoe time, while $n=500$ is a number of echoes. In addition, $T_{1}$ encoding has been performed with $T_{s}$ ranging from 0.1 to $4000 \mathrm{~ms}$, according to a geometric progression, with a common ratio of 1.3 and with 41 different values; the number of scans per each $\left(I_{s}\right)$ were 1024 . Data have been processed by means of the 2D Fast Laplace inversion algorithm [57], which calculates the distribution functions $P\left(T_{1}, T_{2}\right)$ of the data from $T_{1}-T_{2}$ pulse sequence. $T_{1}-T_{2}$ maps of the $L_{1}-L_{12}$ sections have been calculated. For a sake of brevity, only the $L_{5}$ and $L_{7}$ maps are shown herein (Figs. 7b,c), which are connected to the areas surrounded by two dotted red rectangles in Fig. 7a. All signals were normalized to the highest value recorded. Coloured contour lines represent different signal intensities with increasing values, as indicated in the legend of Fig. 7. Maps illustrate the presence of two different sites for water molecules: one with the highest $T_{2}$ values, associable to more mobile water molecules, and one with the lowest $T_{2}$ values. Indeed, $T_{1}-T_{2}$ maps represent the typical structure of a paper material [58], in which the lowest $\mathrm{T}_{2}$ values correspond to water molecules confined between crystalline and amorphous domains, while the highest $T_{2}$ values represent water molecules in the amorphous domains like water pools [59]. Therefore, NMR data give us information about the cellulosic layer positioned inside the book binding (Fig. 1c). In all maps, $\mathrm{T}_{1}$ and $\mathrm{T}_{2}$ distributions are very similar, but in sections $L_{5}$ and $L_{7}($ Fig. $7 b, c)$ they have higher intensities shown by purple lines. This increase in 


\subsection{1/qirt.2016.119}

intensity could be due to a greater presence of water; also in this case, it is possible to find a link with the position of the anomalies detected in the Kurtogram segmented by a specific algorithm (Fig. 4b). As water molecules preferably occupy amorphous regions in paper, these different intensities may be the effect of biological/chemical degradation events that led to a progressive modification from crystalline into amorphous domains and/or to a growth of water pools.

\section{Conclusions}

The integrated approach provides the possibility to map the most important parts of an ancient book, i.e., the lateral hinge, the front facet and the extremities. In addition, it also characterizes the nature of the defects as presence of water, thanks to the use of the NMR technique. The work introduces many innovations in this field of research. Among these, and to the best of our knowledge:

the integrated use of WTT and coherence analysis in the cultural heritage field,

the corrections of the specklegrams by dark master and flat field master, and the demonstration that this procedure is fundamental before the application of the MatPIV package, useful for the correlation analysis,

the good correlation between the Kurtogram result combined with a segmentation algorithm, and the NMR results, in order to detect the weaker areas of the book binding damaged by the aging effect,

- the first application of the holographic interferometry for the defect detection in book bindings and the interesting correlation with the Kurtogram result, i.e., one of the HOST techniques,

- $\quad$ the subtraction between reflectograms and the transmittograms recorded into the NIR region, in order to improves the detectability of holes linked to the presence of pests (probably, woodworms).

A perspective of the work will be the repetition of the NMR analysis by working with a $100 \%$ U.R. in order to confirm the preliminary results shown herein.

\section{REFERENCES}

[1] Bicchieri M., Pinzari F., Discoveries and oddities in library materials, Microchem J - Vol. 124, pp. 568-577, 2016.

[2] Ershad-Langroudi A., Mirmontahai A., Thermal analysis on historical leather bookbinding treated with PEG and hydroxyapatite nanoparticles, J Therm Anal Calorim - Vol. 120, no. 2, pp. 1119-1127, 2015.

[3] Salerno E., Martinelli F., Tonazzini A., Nonlinear model identification and see-through cancelation from recto-verso documents, Int. J. on Document Analysis and Recognition - Vol. 16, pp. 177-187, 2013.

[4] Petrucci Nardelli F., "Legatura e scrittura: testi celati, messaggi velati, annunci palesi”. L.S. Olschki, pp. 1-4, Firenze, 2007.

[5] Salerno E., Tonazzini A., Bedini L., Digital image analysis to enhance underwritten text in the Archimedes palimpsest, J. on Document Analysis and Recognition - Vol. 9, pp. 79-87, 2007.

[6] Tonazzini A., Savino P., Salerno E., A non-stationary density model to separate overlapped texts in degraded documents, Signal Image and Video Processing - Vol. 9, no. 1, pp. 155-164, 2015.

[7] Micheli L., Mazzuca C., Palleschi A., Palleschi G., Development of a diagnostic and cleaning tool for paper artworks: a case of study, Microchem J - Vol. 126, pp. 32-41, 2016.

[8] Scheper K., "The technique of Islamic bookbinding - Methods, Materials and Regional Varieties". Brill, pp. 1-427, Boston, 2015.

[9] Mercuri F., Zammit U., Orazi N., Paoloni S., Marinelli M., Scudieri F., Active infrared thermography applied to the investigation of art and historic artefacts, J Them Anal Calorim - Vol. 104, pp. 475-485, 2011.

[10] Mercuri F., Orazi N., Zammit U., Paoloni S., Marinelli M., Valentini P.P., Thermographic analysis of cultural heritage: recent applications and perspectives, e-PS - Vol. 9, pp. 84-89, 2012.

[11] Marinelli M., Mercuri F., Scudieri F., Zammit U., Colombo G., Thermographic study of microstructural defects in deteriorated parchment sheets, J Phys IV - Vol. 125, pp. 527-529, 2005.

[12] Colombo G., Mercuri F., Scudieri F., Zammit U., Marinelli M., Volterri R., Thermographic analysis of parchment bindings, Restaurator - Vol. 26, pp. 92-104, 2005.

[13] Mercuri F., Gnoli R., Paoloni S., Orazi N., Zammit U., Cicero C., Marinelli M., Scudieri F., Hidden text detection by infrared thermography, Restaurator - Vol. 34, no. 3, pp. 195-211, 2013.

[14] Sfarra S., Ibarra-Castanedo C., Tortora M., Arrizza L., Cerichelli G., Nardi I., Maldague X., Diagnostics of wall paintings: a smart and reliable approach, J Cult Herit - Vol. 18, pp. 229-241, 2016.

[15] Sfarra S., Bendada A., Ibarra-Castanedo C., Ambrosini D., Paoletti D., Maldague X., Santa Maria di Collemaggio Church (L'Aquila, Italy): historical reconstruction by non-destructive testing techniques, Int J Arch Herit - Vol. 9, pp. 367-390, 2015.

[16] Maldague X.P.V., "Theory and practice of infrared thermography for nondestructive testing". John Wiley \& Sons, $1^{\text {st }}$ Ed., pp. 1 684, New York, 2001.

[17] Madruga F.J., Ibarra-Castanedo C., Conde O.M., López-Higuera J.M., Maldague X., Infrared thermography processing based on higher-order statistics, NDT\&E Int - Vol. 43, no. 8, pp. 661-666, 2010.

[18] Vavilov V.P., Shiryaev V.V., Khorev V.S., Processing of active thermal nondestructive testing results by the method of wavelet analysis, Russ J Nondestruct+ - Vol. 47, no. 4, pp. 276-283, 2011.

[19] Sfarra S., Regi M., Santulli C., Sarasini F., Tirillò J., Perilli S., An innovative nondestructive perspective for the prediction of the effect of environmental aging on impacted composite materials, Int J Eng Sci - Vol. 102, pp. 55-76, 2016.

[20] Georges M.P., Vandenrijt J.-F.., Thizy C., Alexeenko I., Pedrini G., Vollheim. B., Lopez I., Jorge I., Rochet J., Osten W. Combined holography and thermography in a single sensor through image-plane holography at thermal infrared wavelengths, Opt Express Vol. 22, no. 21, pp. 25517-25529, 2014.

[21] Ibarra-Castanedo C., Sfarra S., Ambrosini D., Paoletti D., Bendada A., Maldague X., Subsurface defect characterization in artworks by quantitative pulsed phase thermography and holographic interferometry, Quantitative Infrared Thermography Journal - Vol. 5, no. 2, pp. 131-149, 2008.

[22] Ibarra-Castanedo C., Sfarra S., Ambrosini D., Paoletti D., Bendada A., Maldague X., Diagnostics of panel paintings using holographic interferometry and pulsed thermography, Quantitative Infrared Thermography Journal - Vol. 7, no. 1, pp. 85-114, 2010. 
[23] Theodorakeas P., Ibarra-Castanedo C., Sfarra S., Avdelidis N.P., Koui M., Maldague X., Paoletti D., Ambrosini D., NDT inspection of plastered mosaics by means of transient thermography and holographic interferometry, NDT\&E Int - Vol. 47, pp. 150-156, 2012.

[24] Sfarra S., Theodorakeas P., Avdelidis N.P., Koui M., Thermographic, ultrasonic and optical methods: a new dimension in veneered wood diagnostics, Russ J Nondestruct+ - Vol. 49, no. 4, pp. 234-250, 2013.

[25] Sfarra S., Ibarra-Castanedo C., Ambrosini D., Paoletti D., Bendada A., Maldague X., Discovering the defects in paintings using non-destructive testing (NDT) techniques and passing through measurements of deformation, J Nondestruct Eval - Vol. 33, pp. 358-383, 2014

[26] Sfarra S., Ibarra-Castanedo C., Ridolfi S., Cerichelli G., Ambrosini D., Paoletti D., Maldague X., Holographic interferometry (HI), Infrared Vision and X-Ray Fluorescence (XRF) spectroscopy for the assessment of painted wooden statues: a new integrated approach, Appl Phys A - Vol. 115, pp. 1041-1056, 2014.

[27] Pinniger D., "Managing pests in paper-based collections", in Preservation advisory centre. Esmée Fairbairn Foundation, $1^{\text {st }}$ Ed., pp. 1-16, London, 2012, accessed on November 11, 2015, http://www.bl.uk/aboutus/stratpolprog/collectioncare/publications/booklets/managing_pests_in_paper-based_collections.pdf.

[28] McDonald P., Korb J., Mitchell J., Monteilhet L.. Surface relaxation and chemical exchange in hydrating cement pastes: A twodimensional NMR relaxation study. Physical Review E, - Vol. 72, no. 1, $011409,2005$.

[29] Blümich B., Anferova S., Sharma S., Segre A.L., Federici C. Degradation of Historical Paper: Nondestructive Analysis by the NMR-MOUSE. Journal of Magnetic Resonance, - Vol. 161, no. 2, pp. 204-209, 2003.

[30] Bendada A., Sfarra S., Ibarra-Castanedo C., Akhloufi M., Caumes J.-P., Pradere C., Batsale J.-C., Maldague X., Subsurface imaging for panel paintings inspection: a comparative study of the ultraviolet, the visible, the infrared and the terahertz spectra, Opto-Electron Rev - Vol. 23, no. 1, pp. 88-99, 2015.

[31] Van Asperen de Boer J.R.J., Infrared reflectograms of panel paintings, Stud Conserv - Vol. 11, pp. 45-46, 1966.

[32] Sfarra S., Ibarra-Castanedo C., Santulli C., Sarasini F., Ambrosini D., Paoletti D., Maldague X., Eco-friendly laminates: from the indentation to non-destructive evaluation by optical and infrared monitoring techniques, Strain - Vol. 49, pp. 175-189, 2013.

[33] Ibarra-Castanedo C., Piau J-M., Guibert S., Maldague X.P., Bendada A. "Chapter 14. Active infrared thermography techniques for the nondestructive testing of materials," in Ultrasonic and Advanced Methods for Nondestructive Testing and Material Characterization, C. H. Chen Ed., p 325- 348, 2006.

[34] Darlington R.B., Is Kurtosis Really Peakedness?, Amer Statist - Vol. 24, pp. 19-22, 1970

[35] Madruga F., Ibarra-Castanedo C., Conde O.M., Maldague X.P., López-Higuera J.M. "Enhanced contrast detection of subsurface defects by pulsed infrared thermography based on the fourth order statistic moment, kurtosis", Proceedings of SPIE 7299, Thermosense XXXI, Burleigh D.D. and Dinwiddie R.B., Eds., Baltimore (USA), 2009.

[36] Torrence C., Compo G.P., A practical guide to wavelet analysis, Bull Am Meteor Soc - Vol. 79, pp. 61-78, 1998.

[37] Torrence C., Webster P., Interdecadal changes in the ESNO-Monsoon System, J Clim - Vol. 12, pp. 2679-2690, 1999.

[38] Mehta P.C., Rampal V.V., "Lasers and holography". World Scientific Publishing Co. Pte. Ltd., pp. 1-682, New York, 1993.

[39] Wang H., Kang Y., Fu D., Zhang Z., "Application of digital speckle correlation method to measure fracture toughness of foil material", Proceedings of SPIE 5058, Optical technology and image processing for fluids and solids diagnostics, Shen G.X., Cha S.S., Chiang F.-P. and Mercer C.R., Eds., Beijing (China), 2002.

[40] Sveen J.K., "MatPIV - the PIV toolbox for Matlab", http://folk.uio.no/jks/matpiv/index2.html, accessed on March 17, 2006.

[41] Bendada A., Sfarra S., Genest M., Paoletti D., Rott S., Talmy E., Ibarra-Castanedo C., Maldague X., How to reveal subsurface defects in Kevlar $^{\circledR}$ composite materials after an impact loading using infrared vision and optical NDT techniques?, Eng Fract Mech - Vol. 108, pp. 195-208, 2013.

[42] Van Nieuwenhove V., De Beenhouwer J., De Carlo F., Mancini L., Marone F., Sijbers J., Dynamic intensity normalization using eigen flat fields in X-ray imaging, Opt Express - Vol. 23, no. 21, pp. 27975-27989, 2015.

[43] Cox J., McDonald P., Gardiner B., A study of water exchange in wood by means of 2D NMR relaxation correlation and exchange, Holzforschung - Vol.64, pp.259-266, 2010.

[44] Banister M., "The craft of bookbinding". Dover Publications, Inc., pp. 1-160, New York, 1975.

[45] Bendix C., "Damaged books". The preservation advisory centre, pp. 1-17, London, 2010.

[46] Maldague X., Marinetti S., Pulse phase infrared thermography, J Appl Phys - Vol. 79, no. 5, pp. 2694-2698, 1996.

[47] Zauner G., Mayr G., Hendorfer G. "Wavelet-based subsurface defect characterization in pulsed phase thermography for nondestructive evaluation", Proceedings of SPIE 7248, Wavelet Applications in Industrial Processing VI, Truchetet F. and Laligant O., Eds., San Jose (USA), 2009.

[48] Las.E.R. Laboratory (LASer \& Electro optical Research in engineering metrology), http://laser.ing.univaq.it/, accessed on March 20, 2016

[49] Sfarra S., Ibarra-Castanedo C., Santulli C., Paoletti D., Maldague X., Monitoring of jute/hemp fiber hybrid laminates by nondestructive testing techniques, Sci Eng Compos Mater - accepted for publication, 2014, doi: 10.1515/secm-2013-0138.

[50] Maldague X., Krapez J.C., Poussart D., Thermographic nondestructive evaluation (NDE): an algorithm for automatic defect extraction in infrared images, IEEE Transactions on Systems, Man, and Cybernetics - Vol. 20, no. 3, pp. 722-725, 1990.

[51] Olsen D., Dou C., Zhang X., Hu L., Kim H., Hildum E., Radiometric calibration for AgCam, Remore Sens., - Vol. 2, pp. 464-477, 2010.

[52] Pratt W.K., "Digital image processing". John Wiley \& Sons, pp. 1-698, New York, 1991.

[53] Ibarra-Castanedo C., Bendada A., Maldague X., "Thermographic image processing for NDT", http://www.ndt.net/article/panndt2007/papers/55.pdf, accessed on March 11, 2006.

[54] Weir C.E., Effect of temperature on the volume of leather and collagen in water, Journal of Research of the National Bureau of Standards - Vol. 41, no. RP1924, pp. 279-285, 1948.

[55] Blümich B., Perlo J., Casanova F., Mobile single-sided NMR, Prog Nucl Mag Res Sp - Vol. 52, no. 4, pp. 197-269, 2008.

[56] Terenzi C., Casieri C., Two-dimensional nuclear magnetic resonance correlation maps as a signature of firing in iron-rich ceramics made from carbonatic raw clays, Appl Clay Sci, - Vol. 53, no. 3, pp. 517-524, 2011.

[57] Hürlimann M.D., Venkataramanan L., Quantitative Measurement of Two-Dimensional Distribution Functions of Diffusion and Relaxation in Grossly Inhomogeneous Fields, J Magn Reson, - Vol. 157, no. 1, pp. 31-42, 2002.

[58] Lepore A., Baccaro S., Casieri C., Cemmi A., De Luca F., Role of water in the ageing mechanism of paper, Chem Phys Lett, - Vol. 531, pp. 206-209, 2012

[59] Capitani D., Proietti N., Ziarelli F., NMR Study of Water-Filled Pores in One of the Most Widely Used Polymeric Material: The Paper, Macromolecules, - Vol. 35, no. 14, pp. 5536-5543, 2002. 